\title{
Some New Generating Functions for $q$-Hahn Polynomials
}

\author{
Yun Zhou $^{1}$ and Qiu-Ming Luo ${ }^{2}$ \\ ${ }^{1}$ Basic Courses Department, Southeast University Chengxian College, Dongda Road, Pukou, Nanjing 210088, China \\ ${ }^{2}$ Department of Mathematics, Chongqing Higher Education Mega Center, Chongqing Normal University, Huxi Campus, \\ Chongqing 401331, China
}

Correspondence should be addressed to Qiu-Ming Luo; luomath2007@163.com

Received 10 April 2014; Accepted 25 May 2014; Published 9 June 2014

Academic Editor: Senlin Guo

Copyright (c) 2014 Y. Zhou and Q.-M. Luo. This is an open access article distributed under the Creative Commons Attribution License, which permits unrestricted use, distribution, and reproduction in any medium, provided the original work is properly cited.

We obtain some new generating functions for $q$-Hahn polynomials and give their proofs based on the homogeneous $q$-difference operator.

\section{Introduction}

Throughout this paper we suppose that $q \in \mathbb{C},|q|<1$, and the $q$-shifted factorials are defined by

$$
\begin{gathered}
(a ; q)_{0}=1, \quad(a ; q)_{n}=\prod_{k=0}^{n-1}\left(1-a q^{k}\right), \\
(a ; q)_{\infty}=\prod_{k=0}^{\infty}\left(1-a q^{k}\right), \quad n \geq 1 .
\end{gathered}
$$

Clearly,

$$
(a ; q)_{n}=\frac{(a ; q)_{\infty}}{\left(a q^{n} ; q\right)_{\infty}}
$$

We also adopt the following compact notation for the multiple $q$-shifted factorials:

$$
\begin{aligned}
\left(a_{1}, a_{2}, \ldots, a_{m} ; q\right)_{n} & =\left(a_{1} ; q\right)_{n}\left(a_{2} ; q\right)_{n} \cdots\left(a_{m} ; q\right)_{n}, \\
\left(a_{1}, a_{2}, \ldots, a_{m} ; q\right)_{\infty} & =\left(a_{1} ; q\right)_{\infty}\left(a_{2} ; q\right)_{\infty} \cdots\left(a_{m} ; q\right)_{\infty} .
\end{aligned}
$$

The basic hypergeometric series or $q$-series ${ }_{r} \phi_{s}$ are defined by

$$
\begin{aligned}
& { }_{r} \phi_{s}\left(\begin{array}{l}
a_{1}, a_{2}, \ldots, a_{r} \\
b_{1}, b_{2}, \ldots, b_{s} ;
\end{array} \quad q, z\right) \\
& =\sum_{n=0}^{\infty} \frac{\left(a_{1}, a_{2}, \ldots, a_{r} ; q\right)_{n}}{\left(q, b_{1}, b_{2}, \ldots, b_{s} ; q\right)_{n}}\left[(-1)^{n} q^{\left(\begin{array}{c}
n \\
2
\end{array}\right)}\right]^{1+s-r} z^{n} .
\end{aligned}
$$

Euler identity is as follows:

$$
\sum_{n=0}^{\infty} \frac{t^{n}}{(q ; q)_{n}}=\frac{1}{(t ; q)_{\infty}}
$$

The $q$-binomial theorem is as follows:

$$
\sum_{n=0}^{\infty} \frac{(a ; q)_{n}}{(q ; q)_{n}} x^{n}=\frac{(a x ; q)_{\infty}}{(x ; q)_{\infty}}
$$

The usual $q$-differential operator or $q$-derivative operator $D_{q}$ is defined by (see [1, Page 177, (2.1)])

$$
\begin{gathered}
D_{q}\{f(a)\}=\frac{f(a)-f(a q)}{a}, \\
D_{q}^{n}\{f(a)\}=D_{q}\left\{D_{q}^{n-1}\{f(a)\}\right\} .
\end{gathered}
$$

In [1], Chen and Liu introduced the $q$-exponential $T\left(b D_{q}\right)$ operator as follows (see [1, Page 17, (2.5)]):

$$
T\left(b D_{q}\right)=\sum_{n=0}^{\infty} \frac{\left(b D_{q}\right)^{n}}{(q ; q)_{n}},
$$


and they get the $q$-operator identity of $T\left(b D_{q}\right)$ (see $[1$, Page 178, Theorems 2.2 and 2.3]) as follows:

$$
\begin{gathered}
T\left(b D_{q}\right)\left\{\frac{1}{(a t ; q)_{\infty}}\right\}=\frac{1}{(a t, b t ; q)_{\infty}} \quad|b t|<1, \\
T\left(b D_{q}\right)\left\{\frac{1}{(a s, a t ; q)_{\infty}}\right\}=\frac{(a b s t ; q)_{\infty}}{(a s, a t, b s, b t ; q)_{\infty}} \quad|b t|<1 .
\end{gathered}
$$

Recently Chen et al. [2] introduced the following homogeneous $q$-difference $D_{x y}$

$$
D_{x y}\{f(x, y)\}=\frac{f\left(x, q^{-1} y\right)-f(q x, y)}{x-q^{-1} y}
$$

and the homogeneous $q$-difference operator $E\left(D_{x y}\right)$ :

$$
E\left(D_{x y}\right)=\sum_{k=0}^{\infty} \frac{D_{x y}^{k}}{(q ; q)_{k}}
$$

They obtained some properties of $D_{x y}$ as follows:

$$
\begin{gathered}
D_{x y}\left\{P_{n}(x, y)\right\}=\left(1-q^{n}\right) P_{n-1}(x, y), \\
D_{x y}\left\{\frac{(y t ; q)_{\infty}}{(x t ; q)_{\infty}}\right\}=t \frac{(y t ; q)_{\infty}}{(x t ; q)_{\infty}} \\
D_{x y}^{k}\left\{\frac{(y t ; q)_{\infty}}{(x t ; q)_{\infty}}\right\}=t^{k} \frac{(y t ; q)_{\infty}}{(x t ; q)_{\infty}} \\
E\left(D_{x y}\right)\left\{\frac{(y t ; q)_{\infty}}{(x t ; q)_{\infty}}\right\}=\frac{(y t ; q)_{\infty}}{(t, x t ; q)_{\infty}} .
\end{gathered}
$$

The classical Rogers-Szegö polynomial is defined by means of the generating function:

$$
\sum_{n=0}^{\infty} h_{n}(x \mid q) \frac{t^{n}}{(q ; q)_{n}}=\frac{1}{(t, x t ; q)_{\infty}}, \quad|t|<1 ;
$$

obviously, we have

$$
T\left(D_{q}\right)\left\{x^{n}\right\}=h_{n}(x \mid q)=\sum_{k=0}^{n}\left[\begin{array}{l}
n \\
k
\end{array}\right] x^{k}
$$

The homogeneous Rogers-Szegö polynomial is defined by

$$
h_{n}(x, y \mid q)=\sum_{k=0}^{n}\left[\begin{array}{l}
n \\
k
\end{array}\right] P_{k}(x, y) \text {, }
$$

where $P_{n}(x, y)=(x-y)(x-y q) \cdots\left(x-y q^{n-1}\right)$. Clearly, $h_{n}(x, y \mid q)=\Phi_{n}^{(y / x)}(x)$ are the Cauchy polynomials with the following generating function:

$$
\sum_{k=0}^{\infty} P_{k}(x, y) \frac{z^{k}}{(q ; q)_{k}}=\frac{(y z ; q)_{\infty}}{(x z ; q)_{\infty}}, \quad|x z|<1 .
$$

From the above properties, we have

$$
\begin{aligned}
& E\left(D_{x y}\right)\left\{P_{n}(x, y)\right\}=h_{n}(x, y \mid q), \\
& \sum_{n=0}^{\infty} h_{n}(x, y \mid q) \frac{t^{n}}{(q ; q)_{n}}=\frac{(y t ; q)_{\infty}}{(t, x t ; q)_{\infty}} .
\end{aligned}
$$

Lemma 1 (see [3, Lemma 2.3]). For $|t|,|x t|<1$,

$$
\begin{aligned}
E\left(D_{x y}\right) & \left\{\frac{(y t ; q)_{\infty}}{(x t ; q)_{\infty}} \frac{P_{n}(x, y)}{(y t ; q)_{n}}\right\} \\
= & \frac{(y t ; q)_{\infty}}{(t, x t ; q)_{\infty}} \sum_{k=0}^{n}\left[\begin{array}{l}
n \\
k
\end{array}\right] \frac{(y, x t ; q)_{k}}{(y t ; q)_{k}} x^{n-k} .
\end{aligned}
$$

$q$-Hahn polynomial is defined by [4]

$$
\sum_{n=0}^{\infty} \Phi_{n}^{(a)}(x) \frac{t^{n}}{(q ; q)_{n}}=\frac{(a x t ; q)_{\infty}}{(t, x t ; q)_{\infty}} .
$$

We have

$$
\Phi_{n}^{(a)}(x)=\sum_{k=0}^{n}\left[\begin{array}{l}
n \\
k
\end{array}\right](a ; q)_{k} x^{k}
$$

Clearly, $\Phi_{n}^{(0)}(x)=h_{n}(x \mid q)$.

Recently, Chen et al. [3] gave some new proofs of the following results based on the method of homogeneous $q$ difference operator $E\left(D_{x y}\right)$.

Theorem 2. Consider the following:

$$
\begin{aligned}
\sum_{n=0}^{\infty} \Phi_{n}^{(a)}(x) \Phi_{n}^{(b)}(y) \frac{t^{n}}{(q ; q)_{n}} & \\
\quad= & \frac{(x a t, y b t ; q)_{\infty}}{(t, x t, y t ; q)_{\infty}} \phi_{2}\left(\begin{array}{c}
t, a, b \\
x a t, y b t ;
\end{array} \quad q, x y t\right)
\end{aligned}
$$

Theorem 3. Consider the following:

$$
\begin{aligned}
\sum_{n=0}^{\infty} & \sum_{m=0}^{\infty} \Phi_{m+n}^{(a)}(x) \frac{t^{n}}{(q ; q)_{n}} \frac{s^{m}}{(q ; q)_{m}} \\
& =\frac{(x a s ; q)_{\infty}}{(s, x s, x t ; q)_{\infty}}{ }_{2} \phi_{1}\left(\begin{array}{cc}
x a, x s \\
x a s ;
\end{array}, q, t\right) .
\end{aligned}
$$
5-16].

In the present paper, we obtain some new generating functions for $q$-Hahn polynomials and give their proofs based on the homogeneous $q$-difference operator.

\section{Some New Generating Functions for $q$-Hahn Polynomial}

In the present section we obtain the following new generating functions of $q$-Hahn polynomial. 
Theorem 4. For $|z|<1$,

$$
\sum_{k=0}^{\infty} \Phi_{n+k}^{(a)}(x) \frac{z^{k}}{(q ; q)_{k}}=\frac{(a x z ; q)_{\infty}}{(z, x z ; q)_{\infty}} \sum_{k=0}^{n}\left[\begin{array}{l}
n \\
k
\end{array}\right] \frac{(a, z ; q)_{k}}{(a x z ; q)_{k}} x^{k}
$$

Proof. Let $x \mapsto y$ and $a \mapsto b$ in (21), we have

$$
\begin{aligned}
\sum_{n=0}^{\infty} \Phi_{n}^{(a)} & (x) \Phi_{n}^{(b)}(y) \frac{z^{n}}{(q ; q)_{n}} \\
& =\sum_{n=0}^{\infty} \Phi_{n}^{(a)}(x) \sum_{k=0}^{n}\left[\begin{array}{l}
n \\
k
\end{array}\right](b ; q)_{k} y^{k} \frac{z^{n}}{(q ; q)_{n}} \\
& =\sum_{k, n=0}^{\infty} \Phi_{n+k}^{(a)}(x) \frac{(b ; q)_{k} z^{n}}{(q ; q)_{n}(q ; q)_{k}}(y z)^{k} .
\end{aligned}
$$

By the $q$-binomial theorem $(6)$ and noting that $(b ; q)_{n+k}=$ $\left(b q^{k} ; q\right)_{n}(b ; q)_{k}$, we have

$$
\begin{aligned}
& \frac{(x a z, y b z ; q)_{\infty}}{(z, x z, y z ; q)_{\infty}} \sum_{k=0}^{\infty} \frac{(a, b, z ; q)_{k}}{(a x z, b y z, q ; q)_{k}}(x y z)^{k} \\
& =\frac{(x a z ; q)_{\infty}}{(z, x z ; q)_{\infty}} \sum_{k=0}^{\infty} \frac{(a, b, z ; q)_{k}}{(a x z, q ; q)_{k}}(x y z)^{k} \frac{\left(b y z q^{k} ; q\right)_{\infty}}{(y z ; q)_{\infty}} \\
& =\frac{(x a z ; q)_{\infty}}{(z, x z ; q)_{\infty}} \sum_{k=0}^{\infty} \frac{(a, b, z ; q)_{k}}{(a x z, q ; q)_{k}}(x y z)^{k} \sum_{n=0}^{\infty} \frac{\left(b q^{k} ; q\right)_{n}}{(q ; q)_{n}}(y z)^{n} \\
& =\frac{(x a z ; q)_{\infty}}{(z, x z ; q)_{\infty}} \sum_{n, k=0}^{\infty} \frac{(a, z ; q)_{k}}{(a x z, q ; q)_{k}} \frac{(b ; q)_{n+k}}{(q ; q)_{n}} x^{k}(y z)^{n+k} .
\end{aligned}
$$

By (17), (25), and (26), we obtain

$$
\begin{aligned}
& \sum_{k, n=0}^{\infty} \Phi_{n+k}^{(a)}(x) \frac{(b ; q)_{k} z^{n}}{(q ; q)_{n}(q ; q)_{k}}(y z)^{k} \\
& \quad=\frac{(x a z ; q)_{\infty}}{(z, x z ; q)_{\infty}} \sum_{n, k=0}^{\infty} \frac{(a, z ; q)_{k}}{(a x z, q ; q)_{k}} \frac{(b ; q)_{n+k}}{(q ; q)_{n}} x^{k}(y z)^{n+k}
\end{aligned}
$$

Comparing the coefficients of $y^{k} /(q ; q)_{k}$ on both sides of (27), we obtain the formula (24) immediately. This proof is complete.

Theorem 5. For $|t|<1$,

$$
\begin{aligned}
& \sum_{n=0}^{\infty} \Phi_{m+n}^{(a)}(x) \Phi_{n}^{(b)}(y) \frac{t^{n}}{(q ; q)_{n}} \\
& =\frac{(x a t ; q)_{\infty}}{(t, x t ; q)_{\infty}} \sum_{k=0}^{\infty} \frac{(b ; q)_{k}(x y t)^{k}}{(q ; q)_{k}} \sum_{j=0}^{m+k}\left[\begin{array}{c}
m+k \\
j
\end{array}\right] \frac{(x a, x t ; q)_{j}}{(x a t ; q)_{j}} x^{m-j} .
\end{aligned}
$$

Proof. By (17) and (19), we have

$$
\begin{aligned}
\sum_{n=0}^{\infty} h_{m+n}(x, y \mid q) h_{n}(u, v \mid q) \frac{t^{n}}{(q ; q)_{n}} \\
=\sum_{n=0}^{\infty} E\left(D_{x y}\right)\left\{P_{m+n}(x, y)\right\} h_{n}(u, v \mid q) \frac{t^{n}}{(q ; q)_{n}} \\
=E\left(D_{x y}\right)\left\{\sum_{n=0}^{\infty} P_{m+n}(x, y) \sum_{k=0}^{n}\left[\begin{array}{l}
n \\
k
\end{array}\right] P_{k}(u, v) \frac{t^{n}}{(q ; q)_{n}}\right\} \\
=E\left(D_{x y}\right)\left\{\sum_{k=0}^{\infty} \sum_{n=0}^{\infty} \frac{P_{m+n+k}(x, y) P_{k}(u, v) t^{n+k}}{(q ; q)_{k}(q ; q)_{n}}\right\} \\
=E\left(D_{x y}\right)\left\{\sum_{k=0}^{\infty} \frac{P_{m+k}(x, y) P_{k}(u, v) t^{k}}{(q ; q)_{k}} \sum_{n=0}^{\infty} \frac{P_{n}\left(x, y q^{m+k}\right) t^{n}}{(q ; q)_{n}}\right\} \\
=E\left(D_{x y}\right)\left\{\sum_{k=0}^{\infty} \frac{P_{m+k}(x, y) P_{k}(u, v) t^{k}}{(q ; q)_{k}} \frac{(y t ; q)_{\infty}}{(x t ; q)_{\infty}(y t ; q)_{m+k}}\right\} \\
=\sum_{k=0}^{\infty} \frac{P_{k}(u, v) t^{k}}{(q ; q)_{k}} E\left(D_{x y}\right)\left\{\frac{(y t ; q)_{\infty} P_{m+k}(x, y)}{(x t ; q)_{\infty}(y t ; q)_{m+k}}\right\} \\
=\sum_{k=0}^{\infty} \frac{P_{k}(u, v) t^{k}}{(q ; q)_{k}} \frac{(y t ; q)_{\infty} \sum^{m+k}[m+x t ; q)_{\infty} j \frac{(y, x t ; q)_{j}}{(y t ; q)_{j}} x^{m+k-j}}{j} \\
=\frac{(y t ; q)_{\infty}}{(t, x t ; q)_{\infty}} \sum_{k=0}^{\infty} \frac{(v / u ; q)_{k}(u t x)^{k}}{(q ; q)_{k}} \\
\quad \times \sum_{j=0}^{m+k}[m+k] \frac{(y, x t ; q)_{j}}{(y t ; q)_{j}} x^{m-j} .
\end{aligned}
$$

Setting $y / x=a, v / u=b, u=y$ in the last sum, we obtain the formula (28) of Theorem 5. This proof is complete.

Theorem 6. For $|l|<1,|s|<1,|t|<1$,

$$
\begin{aligned}
\sum_{m, n, k=0}^{\infty} \Phi_{m+k}^{(a)}(x) \Phi_{n+k}^{(b)}(y) \frac{l^{m} s^{n} t^{k}}{(q ; q)_{m}(q ; q)_{n}(q ; q)_{k}} \\
=\frac{(x a l, y b s ; q)_{\infty}}{(l, x l, s, y s ; q)_{\infty}} \sum_{k=0}^{\infty} \frac{t^{k}}{(q ; q)_{k}} \\
\quad \times \sum_{i, j=0}^{\infty}\left[\begin{array}{c}
k \\
i
\end{array}\right]\left[\begin{array}{c}
k \\
j
\end{array}\right] \frac{(x a, x l ; q)_{i}}{(x a l ; q)_{i}} \frac{(y b, y s ; q)_{j}}{(y b s ; q)_{j}} x^{k-i} y^{k-j} .
\end{aligned}
$$

Proof. By (17) and (19), we have

$$
\begin{gathered}
\sum_{m, n, k=0}^{\infty} h_{m+k}(x, y \mid q) h_{n+k}(u, v \mid q) \\
\times \frac{l^{m} s^{n} t^{k}}{(q ; q)_{m}(q ; q)_{n}(q ; q)_{k}}
\end{gathered}
$$




$$
\begin{aligned}
& =\sum_{m, n, k=0}^{\infty} E\left(D_{x y}\right)\left\{P_{m+k}(x, y)\right\} E\left(D_{u v}\right)\left\{P_{n+k}(u, v)\right\} \\
& \times \frac{l^{m} s^{n} t^{k}}{(q ; q)_{m}(q ; q)_{n}(q ; q)_{k}} \\
& =E\left(D_{x y}\right) E\left(D_{u v}\right)\left\{\sum_{m, n, k=0}^{\infty} \frac{P_{m+k}(x, y) P_{n+k}(u, v) l^{m} s^{n} t^{k}}{(q ; q)_{m}(q ; q)_{n}(q ; q)_{k}}\right\} \\
& =E\left(D_{x y}\right) E\left(D_{u v}\right)\left\{\sum_{k=0}^{\infty} \frac{P_{k}(x, y) P_{k}(u, v) t^{k}}{(q ; q)_{k}}\right. \\
& \left.\times \sum_{m=0}^{\infty} \frac{P_{m}\left(x, y q^{k}\right) l^{m}}{(q ; q)_{m}} \sum_{n=0}^{\infty} \frac{P_{n}\left(u, v q^{k}\right) s^{n}}{(q ; q)_{n}}\right\} \\
& =E\left(D_{x y}\right) E\left(D_{u v}\right)\left\{\sum_{k=0}^{\infty} \frac{P_{k}(x, y) P_{k}(u, v) t^{k}}{(q ; q)_{k}}\right. \\
& \left.\times \frac{\left(y l q^{k} ; q\right)_{\infty}}{(x l ; q)_{\infty}} \frac{\left(v s q^{k} ; q\right)_{\infty}}{(u s ; q)_{\infty}}\right\} \\
& =\sum_{k=0}^{\infty} E\left(D_{x y}\right)\left\{\frac{(y l ; q)_{\infty} P_{k}(x, y)}{(x l ; q)_{\infty}(y l ; q)_{k}}\right\} \\
& \times E\left(D_{u v}\right)\left\{\frac{(v s ; q)_{\infty} P_{k}(u, v)}{(u s ; q)_{\infty}(v s ; q)_{k}}\right\} \frac{t^{k}}{(q ; q)_{k}} \\
& =\sum_{k=0}^{\infty}\left\{\frac{(y l ; q)_{\infty}}{(l, x l ; q)_{\infty}} \sum_{i=0}^{k}\left[\begin{array}{c}
k \\
i
\end{array}\right] \frac{(y, x l ; q)_{i}}{(y l ; q)_{i}} x^{k-i}\right\} \\
& \times\left\{\frac{(v s ; q)_{\infty}}{(s, u s ; q)_{\infty}} \sum_{j=0}^{k}\left[\begin{array}{c}
k \\
j
\end{array}\right] \frac{(v, u s ; q)_{j}}{(v s ; q)_{j}} u^{k-j}\right\} \frac{t^{k}}{(q ; q)_{k}} \\
& =\frac{(y l, v s ; q)_{\infty}}{(l, s, x l, u s ; q)_{\infty}} \\
& \times \sum_{k=0}^{\infty} \sum_{i, j=0}^{k}\left[\begin{array}{c}
k \\
i
\end{array}\right]\left[\begin{array}{c}
k \\
j
\end{array}\right] \frac{(y, x l ; q)_{i}(v, u s ; q)_{j} x^{k-i} u^{k-j} t^{k}}{(y l ; q)_{i}(v s ; q)_{j}(q ; q)_{k}} .
\end{aligned}
$$

Setting $y / x=a, v / u=b, u=y$ in the last sum, we obtain the formula (30) of Theorem 6 . This proof is complete.

Theorem 7. For $|t|<1$,

$$
\begin{aligned}
\sum_{k=0}^{\infty} \Phi_{m+k}^{(a)}(x) \Phi_{n+k}^{(b)}(y) \frac{t^{k}}{(q ; q)_{k}} & \\
= & \sum_{k=0}^{\infty} \frac{t^{k}}{(q ; q)_{k}} \sum_{i, j=0}^{\infty}\left[\begin{array}{c}
k \\
i
\end{array}\right]\left[\begin{array}{c}
k \\
j
\end{array}\right](x a ; q)_{i}(b y ; q)_{j} \\
& \times x^{k-i} y^{k-j} \Phi_{m}^{(a)}\left(x q^{i}\right) \Phi_{n}^{(b)}\left(y q^{j}\right) .
\end{aligned}
$$

Proof. Applying (2) and the Euler identity (5) and noting (21), then the right-hand side is equal to (30) as follows:

$$
\begin{aligned}
\frac{(x a l, y b s ; q)_{\infty}}{(l, x l, s, y s ; q)_{\infty}} \sum_{k=0}^{\infty} \frac{t^{k}}{(q ; q)_{k}} & \begin{aligned}
\infty \\
\times
\end{aligned} \sum_{i, j=0}\left[\begin{array}{c}
k \\
i
\end{array}\right]\left[\begin{array}{c}
k \\
j
\end{array}\right] \frac{(x a, x l ; q)_{i}}{(x a l ; q)_{i}} \frac{(y b, y s ; q)_{j}}{(y b s ; q)_{j}} x^{k-i} y^{k-j} \\
= & \sum_{k=0}^{\infty} \frac{t^{k}}{(q ; q)_{k}} \sum_{i, j=0}^{k}\left[\begin{array}{c}
k \\
i
\end{array}\right]\left[\begin{array}{c}
k \\
j
\end{array}\right](x a ; q)_{i}(y b ; q)_{j} x^{k-i} y^{k-j} \\
& \times \frac{\left(x a l q^{i}, y b s q^{j} ; q\right)_{\infty}}{\left(l, s, x l q^{i}, y s q^{j} ; q\right)_{\infty}} \\
= & \sum_{k=0}^{\infty} \frac{t^{k}}{(q ; q)_{k}} \sum_{i, j=0}^{k}\left[\begin{array}{c}
k \\
i
\end{array}\right]\left[\begin{array}{c}
k \\
j
\end{array}\right](x a ; q)_{i}(y b ; q)_{j} x^{k-i} y^{k-j} \\
& \times \sum_{u, v, m, n=0}^{\infty} \frac{(a ; q)_{m}(b ; q)_{n}\left(x l q^{i}\right)^{m}\left(y s q^{j}\right)^{n} l^{u} s^{v}}{(q ; q)_{m}(q ; q)_{n}(q ; q)_{u}(q ; q)_{v}} \\
= & \sum_{k=0}^{\infty} \frac{t^{k}}{(q ; q)_{k}} \sum_{k, j=0}^{k}\left[\begin{array}{c}
k \\
i
\end{array}\right]\left[\begin{array}{c}
k \\
j
\end{array}\right](x a ; q)_{i}(y b ; q)_{j} x^{k-i} y^{k-j} \\
& \times \sum_{u, v, m, n=0}^{\infty} \frac{(a ; q)_{m}(b ; q)_{n}\left(x l q^{i}\right)^{m}\left(y s q^{j}\right)^{n}}{(q ; q)_{m}(q ; q)_{n}(q ; q)_{u}(q ; q)_{v}} l^{m+u} s^{n+v} .
\end{aligned}
$$

By (30) and (33), we have

$$
\begin{aligned}
& \sum_{m, n, k=0}^{\infty} \Phi_{m+k}^{(a)}(x) \Phi_{n+k}^{(b)}(y) \frac{l^{m} s^{n} t^{k}}{(q ; q)_{m}(q ; q)_{n}(q ; q)_{k}} \\
& =\sum_{k=0}^{\infty} \frac{t^{k}}{(q ; q)_{k}} \sum_{i, j=0}^{k}\left[\begin{array}{c}
k \\
i
\end{array}\right]\left[\begin{array}{c}
k \\
j
\end{array}\right](x a ; q)_{i}(y b ; q)_{j} x^{k-i} y^{k-j} \\
& \quad \times \sum_{u, v, m, n=0}^{\infty} \frac{(a ; q)_{m}(b ; q)_{n}\left(x l q^{i}\right)^{m}\left(y s q^{j}\right)^{n}}{(q ; q)_{m}(q ; q)_{n}(q ; q)_{u}(q ; q)_{v}} l^{m+u} s^{n+v} .
\end{aligned}
$$

Comparing the coefficients of $l^{m} s^{n} /(q ; q)_{m}(q ; q)_{n}$ on both sides of (34), we obtain the formula (32) immediately.

Theorem 8. For $|t|<1$,

$$
\begin{aligned}
& \sum_{n=0}^{\infty} \Phi_{m+n}^{(a)}(x) \Phi_{n}^{(b)}(y) \frac{t^{n}}{(q ; q)_{n}} \\
& =\frac{(x y a t, x y b t ; q)_{\infty}}{(x y t, x t, y t ; q)_{\infty}} \\
& \quad \times \sum_{s=0}^{m}\left[\begin{array}{c}
m \\
s
\end{array}\right](a ; q)_{s} x^{s} \frac{(y t ; q)_{s}}{(x y a t ; q)_{s}} \\
& \quad \times{ }_{3} \phi_{2}\left(\begin{array}{c}
x y t, x a, y b \\
x y a t q^{s}, x y b t ;
\end{array} \quad q, t q^{s}\right) .
\end{aligned}
$$


Proof. Set $n=0$ and then let $k \mapsto n$ in (32) and note that $\Phi_{0}^{(b)}(x)=1$; by (21) and (22), we obtain

$$
\begin{aligned}
& \sum_{n=0}^{\infty} \Phi_{m+n}^{(a)}(x) \Phi_{n}^{(b)}(y) \frac{t^{n}}{(q ; q)_{n}} \\
& =\sum_{n=0}^{\infty} \frac{t^{n}}{(q ; q)_{n}} \sum_{i, j=0}^{n}\left[\begin{array}{c}
n \\
i
\end{array}\right]\left[\begin{array}{c}
n \\
j
\end{array}\right](x a ; q)_{i} \\
& \times(y b ; q)_{j} x^{n-i} y^{n-j} \Phi_{m}^{(a)}\left(x q^{i}\right) \\
& =\sum_{n=0}^{\infty} \frac{t^{n}}{(q ; q)_{n}} \sum_{i, j=0}^{n}\left[\begin{array}{c}
n \\
i
\end{array}\right]\left[\begin{array}{c}
n \\
j
\end{array}\right](x a ; q)_{i}(y b ; q)_{j} x^{n-i} y^{n-j} \\
& \times \sum_{s=0}^{m}\left[\begin{array}{c}
m \\
s
\end{array}\right](a ; q)_{s}\left(x q^{i}\right)^{s} \\
& =\sum_{n=0}^{\infty} \frac{t^{n}}{(q ; q)_{n}} \sum_{s=0}^{m}\left[\begin{array}{c}
m \\
s
\end{array}\right](a ; q)_{s} y^{n} \\
& \times \sum_{i=0}^{n}\left[\begin{array}{c}
n \\
i
\end{array}\right](a x ; q)_{i} x^{s+n}\left(\frac{q^{s}}{x}\right)^{i} \sum_{j=0}^{n}\left[\begin{array}{c}
n \\
j
\end{array}\right](b y ; q)_{j}\left(\frac{1}{y}\right)^{j} \\
& =\sum_{n=0}^{\infty} \frac{t^{n}}{(q ; q)_{n}} \sum_{s=0}^{m}\left[\begin{array}{c}
m \\
s
\end{array}\right](a ; q)_{s} x^{s+n} y^{n} \Phi_{n}^{(x a)}\left(\frac{q^{s}}{x}\right) \Phi_{n}^{(y b)}\left(\frac{1}{y}\right) \\
& =\sum_{s=0}^{m}\left[\begin{array}{c}
m \\
s
\end{array}\right](a ; q)_{s} x^{s} \sum_{n=0}^{\infty} \Phi_{n}^{(x a)}\left(\frac{q^{s}}{x}\right) \Phi_{n}^{(y b)}\left(\frac{1}{y}\right) \frac{(x y t)^{n}}{(q ; q)_{n}} \\
& =\sum_{s=0}^{m}\left[\begin{array}{c}
m \\
s
\end{array}\right](a ; q)_{s} x^{s} \frac{\left(x y t a q^{s}, x y b t ; q\right)_{\infty}}{\left(x y t, y t q^{s}, x t ; q\right)^{\infty}} \\
& \times{ }_{3} \phi_{2}\left(\begin{array}{c}
x y t, x a, y b \\
x y a t q^{s}, x y b t ;
\end{array} \quad q, t q^{s}\right) \\
& =\frac{(x y a t, x y b t ; q)_{\infty}}{(x y t, x t, y t ; q)_{\infty}} \sum_{s=0}^{m}\left[\begin{array}{c}
m \\
s
\end{array}\right](a ; q)_{s} x^{s} \frac{(y t ; q)_{s}}{(x y a t ; q)_{s}} \\
& \times{ }_{3} \phi_{2}\left(\begin{array}{c}
x y t, x a, y b \\
x y a t q^{s}, x y b t ;
\end{array} \quad q, t q^{s}\right) .
\end{aligned}
$$

This proof is complete.

\section{Conflict of Interests}

The authors declare that there is no conflict of interests regarding the publication of this paper.

\section{Acknowledgments}

The present investigation was supported by the Natural Science Foundation Project of Chongqing, China, under Grant CSTC2011JJA00024, the Research Project of Science and Technology of Chongqing Education Commission, China, under Grant KJ120625, and the Fund of Chongqing Normal University, China, under Grant nos. 10XLR017 and 2011XLZ07.

\section{References}

[1] W. Y. C. Chen and Z.-G. Liu, "Parameter augmentation for basic hypergeometric series. II," Journal of Combinatorial Theory. Series A, vol. 80, no. 2, pp. 175-195, 1997.

[2] W. Y. C. Chen, A. M. Fu, and B. Zhang, "The homogeneous qdifference operator," Advances in Applied Mathematics, vol. 31, no. 4, pp. 659-668, 2003.

[3] W. Y. C. Chen, H. L. Saad, and L. H. Sun, "The bivariate RogersSzegö polynomials," Journal of Physics A: Mathematical and Theoretical, vol. 40, no. 23, pp. 6071-6084, 2007.

[4] W. Hahn, "Über Polynome, die gleichzeitig zwei verschiedenen Orthogonalsystemen angehören," Mathematische Nachrichten, vol. 2, pp. 263-278, 1949.

[5] D. Bowman, " $q$-difference operators, orthogonal polynomials, and symmetric expansions," Memoirs of the American Mathematical Society, vol. 159, no. 757, 2002.

[6] J. Cao, "A note on moment integrals and some applications," Journal of Mathematical Analysis and Applications, vol. 410, no. 1, pp. 348-360, 2014.

[7] J. Cao, "A note on $q$-integrals and certain generating functions," Studies in Applied Mathematics, vol. 131, no. 2, pp. 105-118, 2013.

[8] J. Cao, "A note on generating functions for Rogers-Szegö polynomials," Quaestiones Mathematicae, vol. 35, no. 4, pp. 447461, 2012.

[9] J. Cao, "Bivariate generating functions for Rogers-Szegö polynomials," Applied Mathematics and Computation, vol. 217, no. 5, pp. 2209-2216, 2010.

[10] J. Cao, "New proofs of generating functions for Rogers-Szegö polynomials," Applied Mathematics and Computation, vol. 207, no. 2, pp. 486-492, 2009.

[11] V. Y. B. Chen and N. S. S. Gu, "The Cauchy operator for basic hypergeometric series," Advances in Applied Mathematics, vol. 41, no. 2, pp. 177-196, 2008.

[12] W. Y. C. Chen and Z.-G. Liu, "Parameter augmentation for basic hypergeometric series. I," in Mathematical Essays in Honor of Gian-Carlo Rota, B. E. Sagan and R. P. Stanley, Eds., vol. 161, pp. 111-129, Birkhäuser, Boston, Mass, USA, 1998.

[13] J.-P. Fang, "q-differential operator identities and applications," Journal of Mathematical Analysis and Applications, vol. 332, no. 2, pp. 1393-1407, 2007.

[14] Z.-G. Liu, "Some operator identities and $q$-series transformation formulas," Discrete Mathematics, vol. 265, no. 1-3, pp. 119139, 2003.

[15] L. J. Rogers, "On the expansion of some infinite products," Proceedings of the London Mathematical Society, vol. 24, no. 1, pp. 337-352, 1893.

[16] H. L. Saad and A. A. Sukhi, "Another homogeneous q-difference operator," Applied Mathematics and Computation, vol. 215, no. 12, pp. 4332-4339, 2010. 


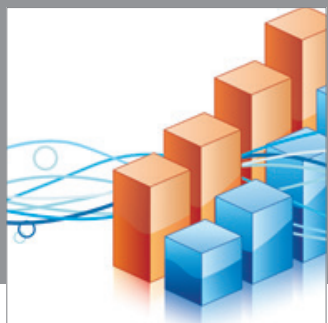

Advances in

Operations Research

mansans

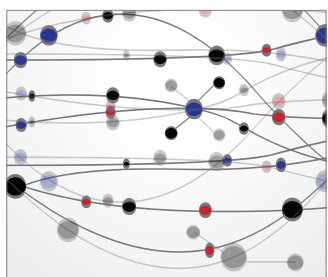

The Scientific World Journal
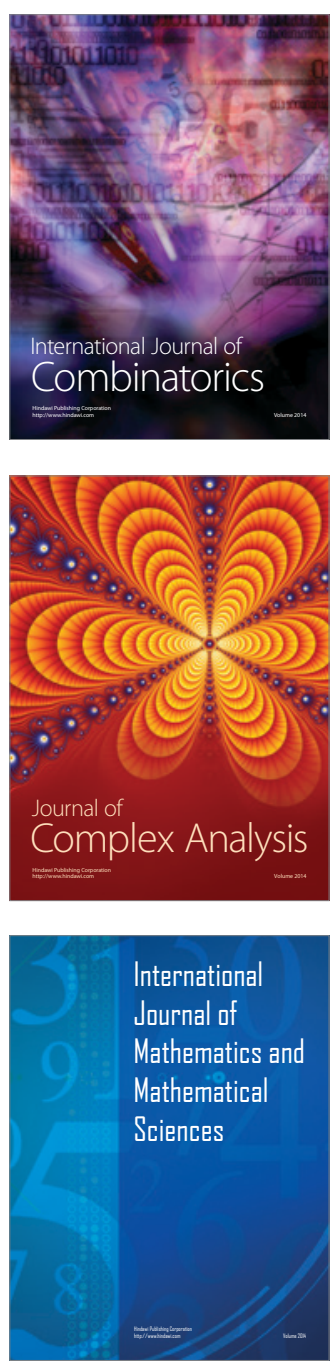
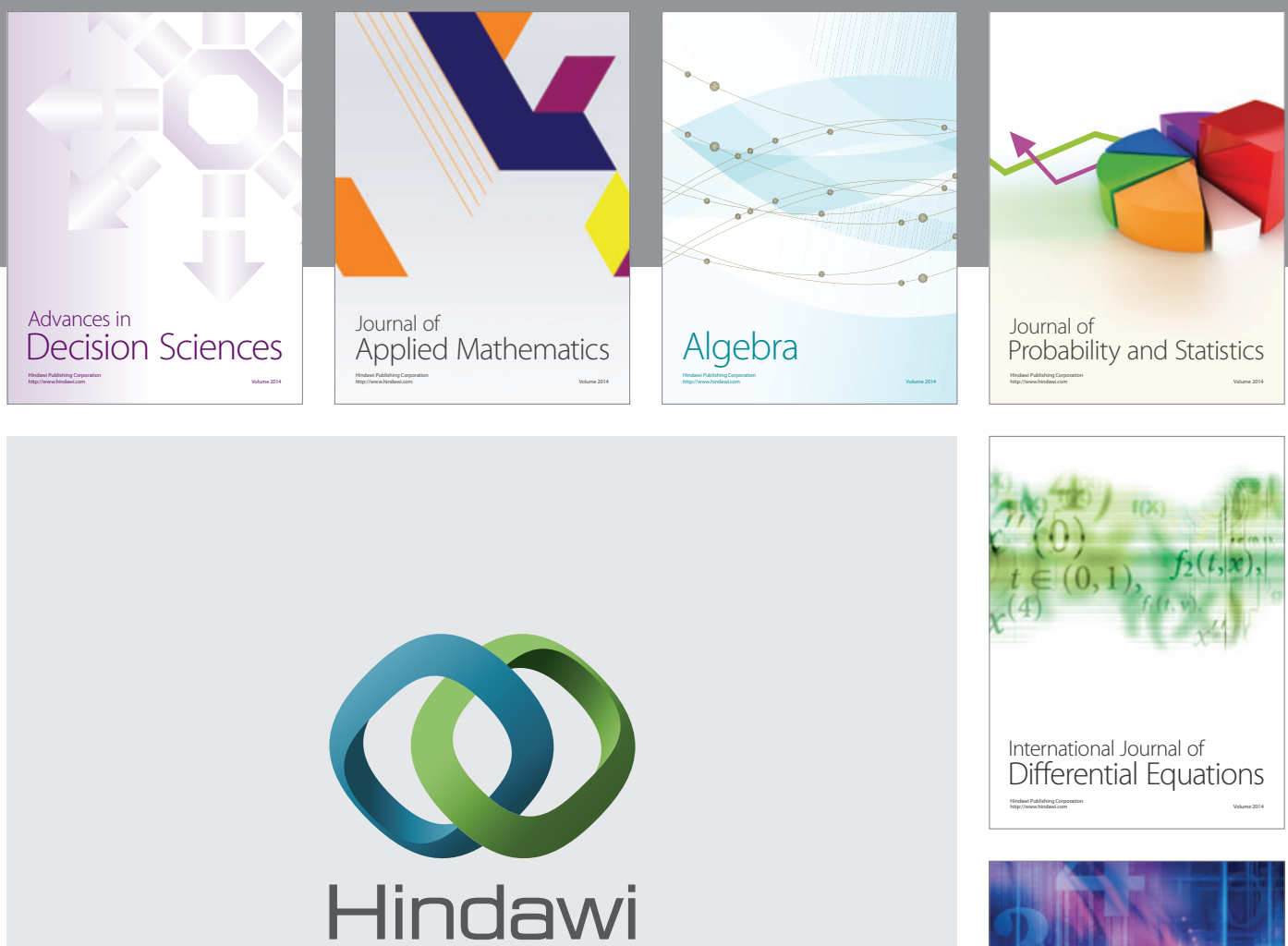

Submit your manuscripts at http://www.hindawi.com
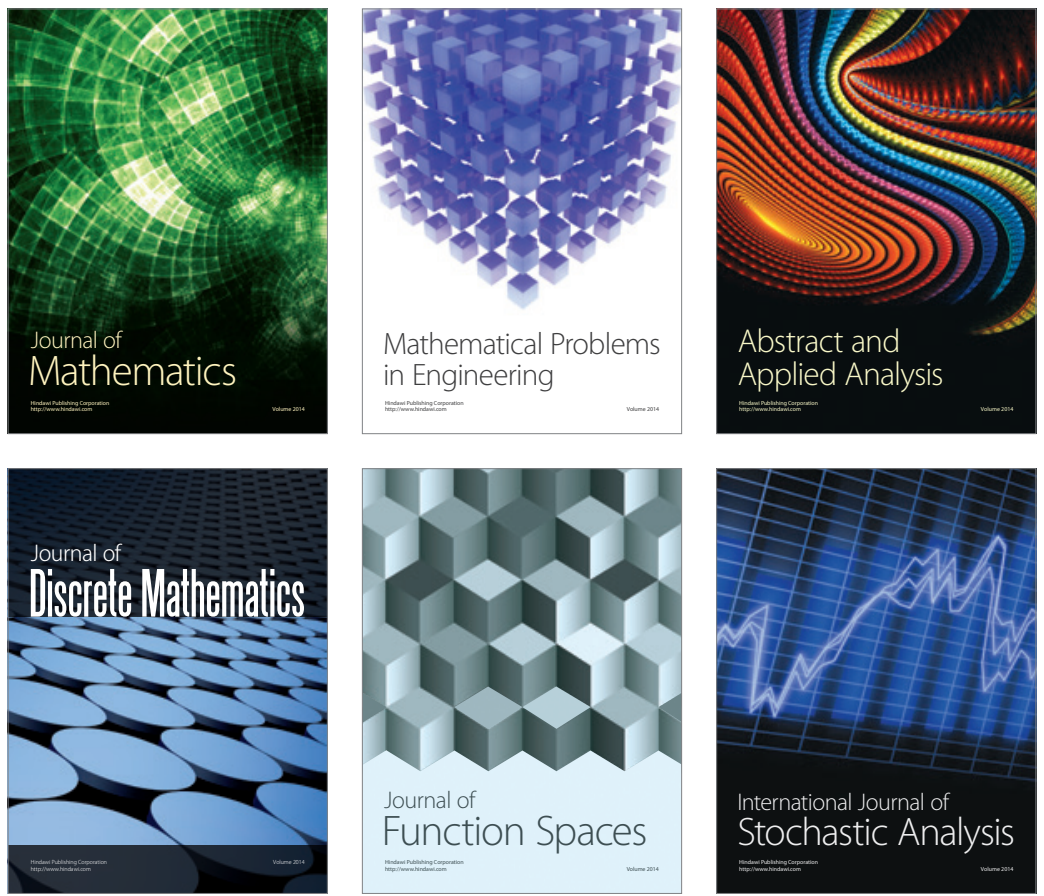

Journal of

Function Spaces

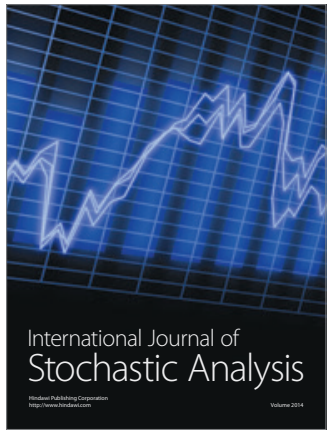

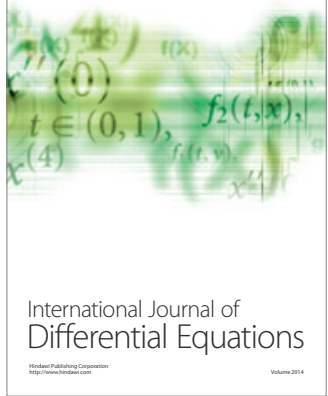
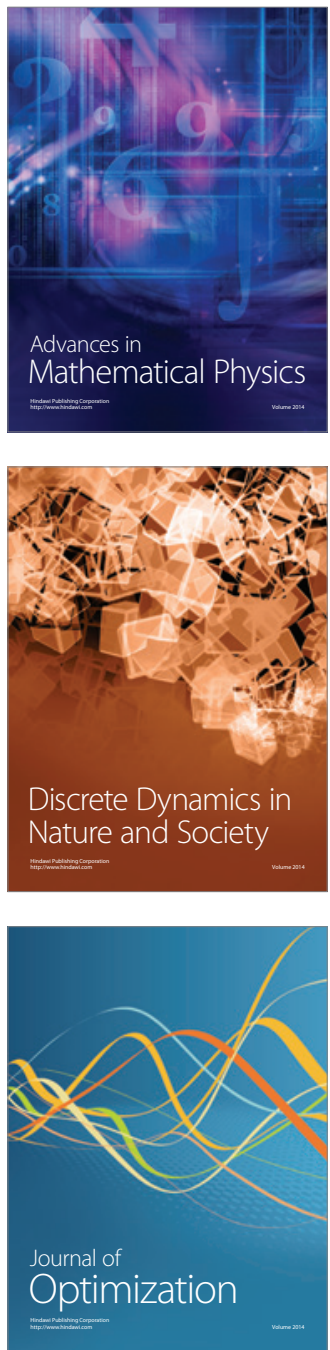